\title{
OFDM FOR WSNS USING CONTINUOUS PHASE MODULATION WITH FRS INTERLEAVER
}

\author{
Fatma H. El-Fouly, ${ }^{*}$ Rabie A. Ramadan, ${ }^{* *}$ and Salah A. Aly***
}

\begin{abstract}
Since wireless sensor networks (WSNs) are characterized by a multipath fading environment, which causes a significant degradation in terms of both network reliability and data rate, the use of orthogonal frequency division multiplexing (OFDM) as a modulation technique is highly needed which is the newest contender against other modulation schemes that provides high quality of service and data rate. The real problem for OFDM implementation in WSNs is the peak to average power ratio (PAPR) of the OFDM signal, which requires highly linear power amplifiers to avoid excessive intermodulation distortion. This is not compatible with the energy conservation which is the major constraint for the design of WSNs. By utilizing continuous phase modulation (CPM) in OFDM system, the PAPR can be effectively reduced to $0 \mathrm{~dB}$. In this paper, we propose to utilize helical interleaving scheme for continuous phase modulation-based orthogonal frequency-division multiplexing (CPM-OFDM) systems. In addition, based on our study to many of the interleavers, we proposed a new interleaver, FRS, that can break the burst channel errors and take the benefits of the previously proposed interleavers, and hence a better bit error rate (BER) performance can be achieved. The proposed system combines the advantages of frequency diversity and power efficiency from CPM-OFDM and performance improvement from new interleaving. Through simulation, the BER performance of the CPM-OFDM system with and without new interleaving is evaluated. In addition, the overall complexity of FRS interleaver compared to helical and 2D-prime interleavers in terms of hardware requirements is evaluated.
\end{abstract}

\section{Key Words}

WSN, CPM-OFDM, FRS interleaver, PAPR

\footnotetext{
* Department of Electronics and Electrical Communication, Higher Institute of Engineering, El-Shorouk Academy, El-Shorouk, Egypt; e-mail: fatma_elfoly@yahoo.com

** Department of Computer Engineering, Faculty of Engineering, Cairo University, Cairo, Egypt; e-mail: rabie@ rabieramadan.org

*** College of Computers and Information Systems, Umm Al-Qura University, Makkah, KSA; e-mail: salah.rid@gmail.com

Recommended by Dr. L. Monticone 85 Dr. K.T. Ma

(DOI: 10.2316/Journal.214.2014.3.214-1076)
}

\section{Introduction}

A wireless sensor network (WSN) is a network of large number of low-cost, battery-operated, multifunctional, and small size nodes. Nodes in this network can monitor the real-world physical conditions or variables (such as temperature and humidity), perform computations, and communicate with each other over a wireless media. They report the collected data to a base station called the "sink" node [1].

WSNs are characterized by a multipath fading environment, which is the main problem faced in communication in wireless networks. In such channels, extreme fading of the signal amplitude occurs and inter-symbol interference (ISI) due to the frequency selectivity of the channel. The fading problem mostly appears at the receiver side. This leads to a significant degradation in terms of both network reliability (due to high probability of errors) and data rate [2].

Orthogonal frequency division multiplexing (OFDM) is considered one of the most effective multicarrier transmission techniques for wireless communications due to its high spectral efficiency obtained by orthogonality, robustness against frequency selective fading, and simple equalizer implementation. At the same time, it has become the widely recognized modulation technique for high data rate communications over wireless links [3]. Despite all of the attractive advantages, one of the major drawbacks of OFDM for low power application is the high peak to average power ratio (PAPR) which requires the use of highly linear power amplifiers. To achieve this linearity, the amplifiers have to be designed with a large back off from their peak power. This results in a very poor power efficiency, which is not compatible with the power consumption reduction and can turn out to be a real problem for OFDM WSN implementation [4].

Continuous phase modulation (CPM) is very attractive for wireless communication due to its constant envelope. This constant envelop is required for efficient power transmission. In addition, CPM is able to exploit the diversity of multipath channel leading to an improvement in the bit error rate (BER) performance. CPM can be used to solve the problems associated with the PAPR of the 
OFDM system [5]. By utilizing CPM in OFDM system, the PAPR can be effectively reduced to $0 \mathrm{~dB}$. Despite CPM features such as low system complexity and favourable performance due to low PAPR and robustness to amplitude variation and impulsive noise, it has low spectral efficiency [6]. At the same the burst errors is another problem in the wireless communication in general.

Another approach that we propose in this paper is the performance enhancement of data transmission in CPMOFDM systems through data interleaving. It is known that data transmission over wireless channel may serve adverse conditions, especially burst errors where errors are likely to occur in clusters. Interleavers have been used extensively in digital communication systems in order to disperse these burst errors [7]-[9]. There are some simple interleaving techniques have been proposed [10], [11]. The performance of such simple interleavers is limited. Therefore, there is a need for more powerful interleavers.

In this paper, a new interleaver, FRS, is proposed in order to achieve better error probability performance. The proposed approach can combat the channel effects without a need for complicated coding schemes for error detection and correction or adaptation in the modulation scheme. Another advantage of the proposed interleaving scheme is that it achieves a degree of encryption in the transmitted data which adds more security to the data transmission process.

The rest of this paper is organized as follows. The OFDM system model and PAPR problem is introduced in Section 2. Then, CPM system is described in Section 3. The problem description is introduced in Section 4. CPMOFDM system model is described in Section 5. Then, Section 6 describes the phase demodulator. The new interleaver, FRS, is described in Section 7. In addition, the proposed system model is elaborated in Section 8. The simulation results are depicted in Section 9. Finally, the conclusions are presented in Section 10.

\section{OFDM and PAPR Problem}

OFDM sends $N$ symbols as a block instead of transmitting data symbols serially. The OFDM block time, $T$ is $N$ times longer than the original symbol time, $T_{s}$ (i.e., $T=N T_{s}$ ). For a block of $N$-modulated data symbols from a particular signaling constellation, $X_{k}=\left(X_{0}, X_{1}, \ldots, X_{N-1}\right)$, over a time interval $[0, T]$, the multicarrier OFDM signal is generated by summing all the $N$-modulated subcarriers each of which is separated by $\nabla f=1 / N T_{s}$ in the frequency domain. The continuous time baseband of OFDM signal is defined by [12], [13] as shown in (1):

$$
X(t)=\frac{1}{\sqrt{N}} \sum_{k=0}^{N-1} X_{k} e^{j 2 \pi f_{k} t} \quad 0 \leq t<T
$$

where $f_{k}=k \nabla f$ is the centre frequency of the $N$ subcarriers. Actually, (1) can be realized conveniently by using the inverse fast Fourier transform (IFFT).

The PAPR of OFDM signal $X(t)$ is defined as the ratio of the peak instantaneous power to the average power of the OFDM transmission, which is expressed in (2) as follows [14]:

$$
\operatorname{PAPR}=\frac{\operatorname{Max}_{0 \leq t<T}\left\{|X(t)|^{2}\right\}}{E\left[|X(t)|^{2}\right]}
$$

where $E[$.$] is the expectation operator. High PAPR$ has been recognized as one of the major practical problem involving OFDM system. High PAPR results from the nature of the modulation itself where multiple subcarrier/sinusoids are added together to form the OFDM signal to be transmitted. When $N$ subcarriers are added with the same phase they produce a peak power that is $N$ times the average power of the signal. Therefore, OFDM signal is considered to have a very large PAPR.

\section{CPM Modulation}

$\mathrm{CPM}$ is a nonlinear modulation scheme in which the information symbols are used to modulate the phase of a carrier signal. The transmitted phase function is continuous over time for all symbol sequences, which makes the envelope of the transmitted signal to be constant. Therefore, non-linear amplifiers are not a problem in this case. Furthermore, the continuous phase function in CPM causes small spectral side lobes as compared to, e.g., the discontinuous phase function in constant amplitude phase shift key (PSK) modulation. The total phase change for each symbol depends on a parameter called the modulation index, $h[15]$.

\section{Problem Description}

In WSN aerospace applications such as aircraft testing, sensor nodes are spread on the aircraft wings to monitor the pressure around the wing during test fight. This WSN will enable aerodynamicists to get a large amount of frequently (every few micro seconds) updated data points. If one measure gets lost, the whole data set recorded during the flight test becomes unusable. Therefore, the reliable and high data rate transmission is extremely required for such application [16]. However, the WSNs constitute a special class of wireless data communication networks. The communications over a wireless channel are usually influenced by multipath fading which causes a significant degradation in terms of both network reliability and data rate. Hence, it is highly needed to use a modulation technique that provides high quality of service and data rate such as OFDM which is the newest contender against other modulation schemes for this purpose.

The real problem for OFDM WSN implementation is the PAPR of the OFDM signal which requires highly linear power amplifiers to avoid excessive intermodulation distortion. To achieve this linearity, the amplifiers have to be designed with a large back off from their peak power. This results in very poor power efficiency. Therefore, OFDM is considered power inefficient. Consequently, this is not compatible with the energy conservation which is the major constraint for the design of WSNs. 

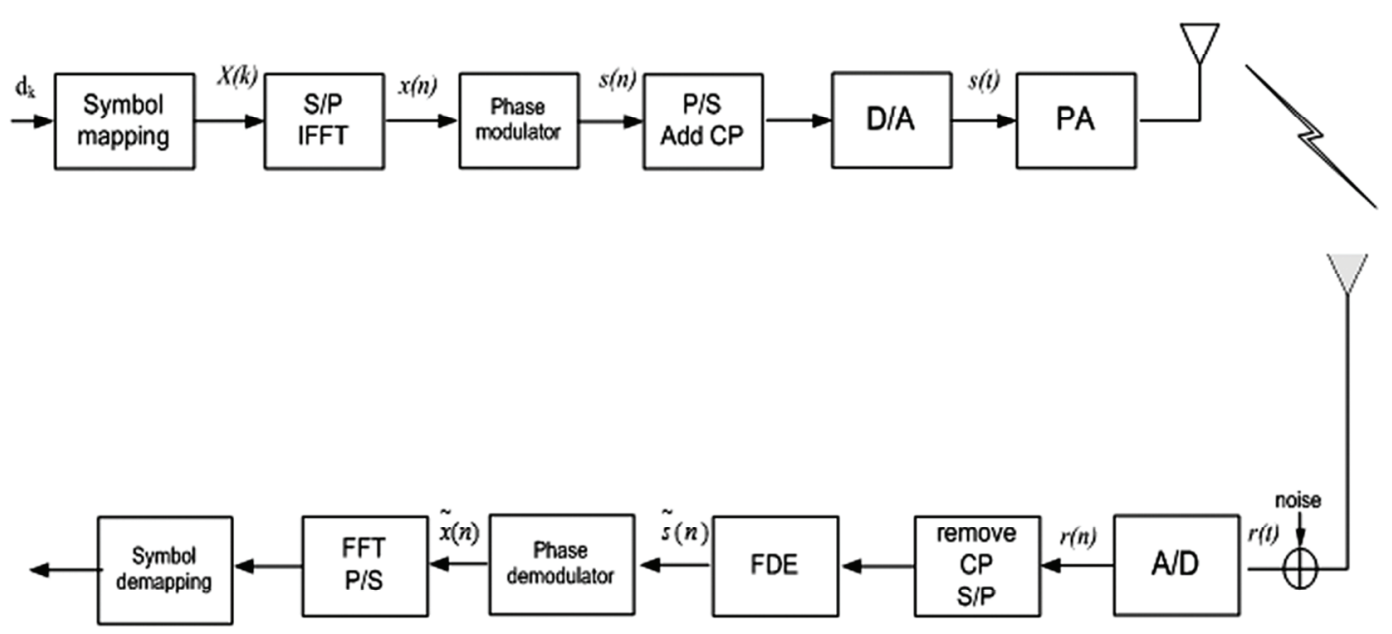

Figure 1. The CPM-OFDM system model [28].

By utilizing CPM in OFDM system, the PAPR can be effectively reduced to $0 \mathrm{~dB}$. Consequently, in this paper, CPM-OFDM system is proposed. The proposed system combines the advantages of OFDM and the energy efficiency of CPM. In addition, some interleavers are proposed to be used with CPM-OFDM system to achieve better performance including helical interleaver. However, the paper proposes a new interleaver entitled "FRS" that proved to be more efficient than the currently used interleavers including helical.

\section{CPM-OFDM Combination}

The CPM-OFDM is a modulation format that can be viewed as a mapping of the OFDM signal onto the unit circle. The instantaneous power of the resulting signal is constant. Therefore, the PAPR is $0 \mathrm{~dB}$. The significance of the $0 \mathrm{~dB}$ PAPR achieved by using CPM is that the signal can be amplified with power efficient nonlinear amplifier [17]-[20]. This is implemented through a straight forward modification of a standard OFDM system as shown in Fig. 1.

In CPM-OFDM transmitter, the input data $\left\{d_{k}\right\}$ is first processed by a symbol mapping. The quadrature phase shift keying (QPSK) modulator is used for this work to map the data to appropriate QPSK symbols $X(k)$ $(k=0,1, \ldots, K-1)$. Then, the symbols are serial to parallel $(\mathrm{S} / \mathrm{P})$ converted and the parallel data symbols are fed into IFFT to generate the multicarrier OFDM signal $x(n) . x(n)$ is the time samples of (1). During each $T$-second block interval, $x(n)$ passes through a phase modulator $(\mathrm{PM})$ to get the constant envelope sequence, $s(n)$. After $\mathrm{PM}$, a cyclic prefix (CP) which is a copy of the last part of each data block is attached to the front of itself to mitigate the inter-block interference (IBI). The $\mathrm{CP}$ must have a longer length than the channel impulse response. Finally, CPM-OFDM continuous-time signal, $s(t)$, is generated at the output of the digital-to-analog (D/A) converter and can be written according to [21], [22] as shown by (3):

$$
s(t)=A e^{j \phi(t)}=A e^{j[2 \pi h m(t)+\vartheta]}
$$

where $A$ is the signal amplitude, $h$ is the modulation index, $\theta$ is an arbitrary phase used to achieve CPM [17], and $m(t)$ is the real-valued message signal given by (4) as follows [23]:

$$
m(t)=C_{\mathrm{norm}} \sum_{k=1}^{K} I_{k} q_{k}(t)
$$

where

$$
I_{k}= \begin{cases}\Re\{X(k)\} & k \leq K / 2 \\ -\Im\{X(k-K / 2)\} & k>K / 2\end{cases}
$$

$I_{k} \in\{ \pm 1, \pm 3, \ldots, \pm M-1\}[23]$ are the $M$-ary pulse amplitude modulation (M-PAM) data symbols, $M$ is the number of constellation points, the orthogonal subcarriers $q_{k}(t)$ must also be real-valued and can be written as follows:

$$
q_{k}(t)= \begin{cases}\cos \left(\frac{2 \pi k t}{T}\right) & k \leq K / 2 \\ \sin \left(\frac{2 \pi[k-K / 2] t}{T}\right) & k>K / 2\end{cases}
$$

In (4), $C_{\text {norm }}$ is a constant used to normalize the variance of the message signal $m(t)$ and consequentially, the variance of the phase signal, $\sigma_{\phi}^{2}=(2 \pi h)^{2}$. This requirement is achieved by setting $C_{\text {norm }}$ as [24] $C_{\text {norm }}=\sqrt{2 / N \sigma_{I}^{2}}$, where $\sigma_{I}^{2}=\left(M^{2}-1\right) / 3$ is the variance of the independent and identically distributed data symbols [24].

The subcarriers are centred at the frequencies $\pm i / T \mathrm{~Hz}$, $I=1,2, \ldots, K / 2$. So, an effective double-sided bandwidth of the message signal is defined as $W=K / T$. The bandwidth of $s(t)$ is lower bounded by $W \mathrm{~Hz}$, and a function of the modulation index. A useful bandwidth expression for CPM-OFDM signal is the root-mean-square (RMS) bandwidth [24], [26] as follows:

$$
B=\max (2 \pi h, 1) W \mathrm{~Hz}
$$


The bit rate is $R=K\left(\log _{2} M\right) / T \mathrm{~b} / \mathrm{s}$, and the bandwidth efficiency is

$$
\eta=\frac{R}{B}=\frac{\log _{2} M}{\max (2 \pi h, 1)} \mathrm{bps} / \mathrm{Hz}
$$

The CPM-OFDM signal $s(t)$ is then transmitted through the multipath channel. The channel impulse response is modeled as a wide-sense stationary uncorrelated scattering (WSSUS) process consisting of $L$ discrete paths [27]:

$$
h(t)=\sum_{l=0}^{L-1} h(l) \delta\left(t-\tau_{l}\right)
$$

where $h(l)$ and $\tau_{l}$ are the channel gain and delay of the $l$ th path, respectively. At the receiver side, the process is reversed to obtain the decoded data. The continuous time received signal $r(t)$ can be expressed as follows:

$$
r(t)=\sum_{l=0}^{L-1} h(l) s\left(t-\tau_{l}\right)+n(t)
$$

where $n(t)$ is a complex additive white Gaussian noise (AWGN) with single-sided power spectral density $N_{0}$. The received signal $r(t)$ is first passed through analog-to-digital (A/D) converter to obtain The $i$ th $(i=0,1, \ldots, J K-1)$ sample of it as follows:

$$
r(i)=\sum_{l=0}^{L J-1} h(l) s(i-l)+n(i)
$$

In Fig. 1, the output of $\mathrm{A} / \mathrm{D}$ is sampled at $t=i T=(J K)$, $J$ is the oversampling factor. Then CP is removed and the frequency domain equalizer (FDE) is proposed. The FDE relies on the existence of a CP guard interval between successive data block. Given that the CP duration is at least that of the channel response, the FDE can perfectly invert the channel. The advantage of the FDE is relatively low complexity [29], [30]. Equalizer filter coefficients are calculated by minimum mean square error (MMSE) or zero force (ZF) [31]. MMSE takes into account the signal-tonoise-ratio (SNR) making an optimum trade-off between channel inversion and noise enhancement [32]. Therefore, MMSE equalizer achieves a significantly lower error compared to ZF equalizer. So it is considered in our work. The equalizer signals are then fed to the phase demodulator (details of which are discussed in Section 2) to obtain the estimate of $x(n)$. Defining $N=J K$, fast Fourier transform (FFT) is applied to convert the signal to frequency domain. Finally, the parallel-to-serial (P/S) converter followed by a symbol de-mapping that yields the received bits.

\subsection{Phase Demodulator}

The details of phase demodulator are discussed in this section. The block diagram is illustrated in Fig. 2.

First, the finite impulse response (FIR) filter is optional, but it has been found that it improves performance effectively. It removes the out-of-band noise and

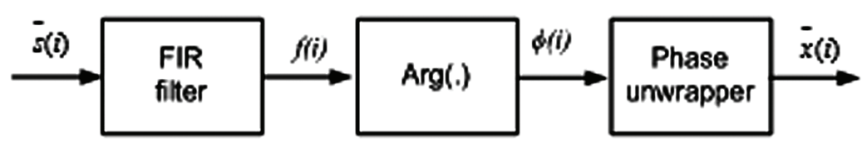

Figure 2. The phase demodulator.

designed using the windowing technique [33]. The filter impulse response with window length $L_{f}$ and a normalized cut-off frequency $f_{\text {nor }}\left(0<f_{\text {nor }} \leq 1\right)$ can be expressed as follows [34]:

$$
g(n)=\frac{\sin \left(2 \pi f_{\text {nor }}\left(n-\frac{L_{f}-1}{2}\right)\right)}{\pi\left(n-\frac{L_{f}-1}{2}\right)}, \quad 0<n \leq L_{f}-1
$$

In (12), if $\left(L_{f}-1\right) / 2, g(n)=2 \pi f_{\text {nor }} / \pi$, the output of the FIR filter can be expressed as follows:

$$
f(i)=\sum_{n=0}^{L_{f}-1} g(n) \tilde{s}(i-n)
$$

Then, $\arg (*)$ operation calculates the phase of the filtered signal $f(i)$ as follows:

$$
\phi(i)=\arg (f(i))=\phi(i)+\delta(i)
$$

where $\phi(i)$ denotes the phase of the desired signal and $\delta(i)$ denotes the phase noise. Then, the phase unwrapper is used to eliminate the effect of any phase ambiguities. As well as, the unwrapper makes the receiver insensitive to phase offsets caused by the channel nonlinearities and/or by the memory terms.

\section{Interleaver}

Due to the fading nature of wireless channels, the bit errors tend to occur in bursts. Most of the error correction codes are designed to correct random errors [34]. Therefore, interleavers have been used extensively in digital communication systems to combat burst errors by converting them into random errors. In general, interleaving rearranges the order of symbols to be transmitted according to a given rule. At the receiver, the reverse rule is used to restore the original sequence [35], [36]. In this section, we focus only on the interleavers which are related to our proposal in this paper. The section starts by explaining helical interleaver followed by $2 \mathrm{D}$-prime interleaver.

\subsection{Helical Interleaver}

The algorithm for helical interleaver generation can be summarized as follows [37], [38]:

1. First generate a primary interleaver by arranging the data sequence in the form of a matrix containing $N_{r}$ rows and $N_{c}$ columns provided that $N_{r} . N_{c}=L$ where $L$ refers to the length of the primary interleaver 


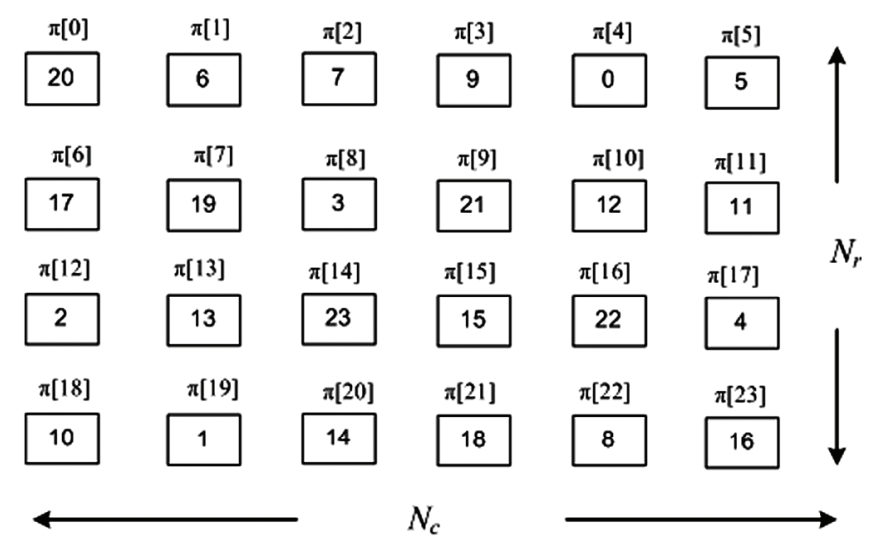

(a)

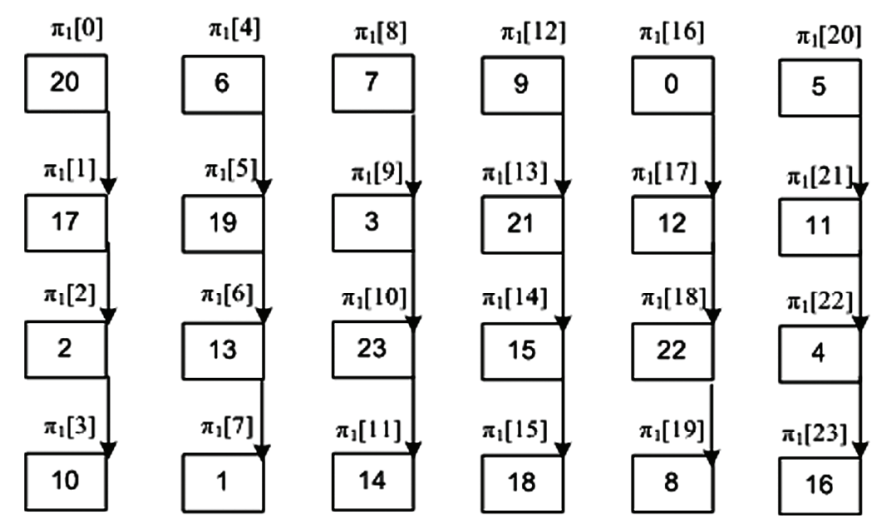

(b)

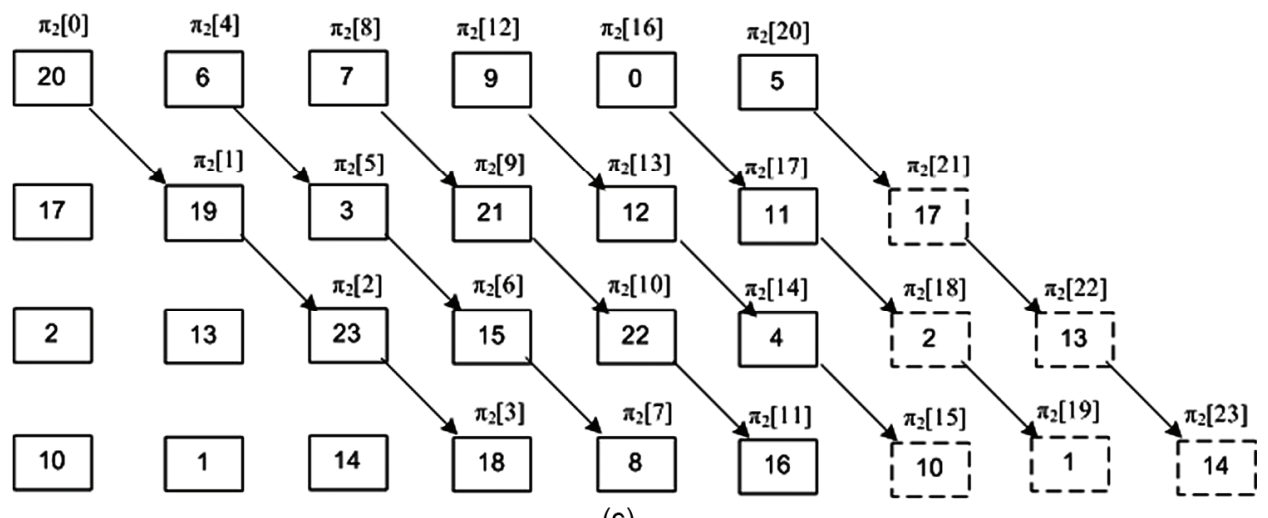

(c)

Figure 3. Generation of $4 \times 6$ helical interleavers: (a) master interleaver written in matrix form, $\pi=[20,6,7, \ldots, 8,16]$; (b) first helical interleaver written in matrix form, $\pi_{1}=[20,17,2, \ldots, 4,16]$; and (c) second helical interleaver written in matrix form, $\pi_{2}=[20,19,23, \ldots, 13,14]$.

which is the same as the number of decoded bits as shown in Fig. 3(a).

2. First helical interleaver is generated from the primary one by reading the interleaver indices column wise as shown in Fig. 3(b).

3. Other interleavers are generated by cyclically reading the interleaver indices from the diagonals of the matrix with decreasing slopes as shown in Fig. 3(c).

A mathematical representation of the $i$ th helical interleaver can be written as

$$
\pi[k]=\pi\left[l_{\bmod L}\right], \quad 0 \leq k<L
$$

where, $l=k_{\bmod N_{r}} \cdot N_{c}+\left(\left[\frac{k}{N_{r}}\right]+\left(k_{\bmod N_{r}} \cdot(i-1)\right)\right)$.

It can be seen from (15) that the generation of helical interleaver is very fast and the interleaver indices can be spread out effectively if the parameters are chosen properly. Now, optimization (15) by introducing layer specific shifts is presented as follows:

$$
\pi[k]=\pi\left\lfloor(l+i . S)_{\bmod N_{c}}\right\rfloor
$$

where, $S$ is a constant integer which describes the shift between the interleavers. 


\subsection{Two-Dimensional Prime Interleaver}

The concept of the 2D-prime interleaver is explained as follows [39]:

1. Consider the case of $N_{r}$ rows and $N_{c}$ columns matrix.

2. Firstly, the interleaving scheme is divided into row-wise interleaving and column-wise interleaving.

3. Secondly, assign two prime numbers as seed to row-wise and column-wise interleavers, respectively. Therefore, the location of bits after interleaving will be as follows:

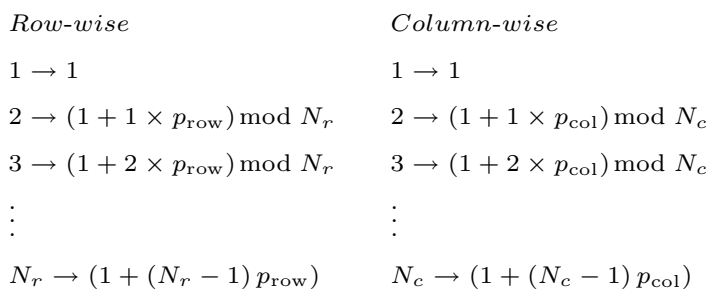

Where $p_{\text {row }}$ and $p_{\text {col }}$ are row-wise and column-wise seeds.

4. Finally, the new locations are mapped back into 2D interleaver to get the resulted interleaved bits in $2 \mathrm{D}$.

For instance, consider $8 \times 8$ 2D-prime interleaver with $p_{\text {row }}=3$ and $p_{\text {col }}=5$. The new locations of bits will be as follows:

$\begin{array}{ll}\text { Row-wise } & \text { Column-wise } \\ 1 \rightarrow 1 & 1 \rightarrow 1 \\ 2 \rightarrow(1+1 \times 3) \bmod 8=4 & 2 \rightarrow(1+1 \times 5) \bmod 8=6 \\ 3 \rightarrow(1+2 \times 3) \bmod 8=7 & 3 \rightarrow(1+2 \times 5) \bmod 8=3 \\ 4 \rightarrow(1+3 \times 3) \bmod 8=2 & 4 \rightarrow(1+3 \times 5) \bmod 8=8 \\ 5 \rightarrow(1+4 \times 3) \bmod 8=5 & 5 \rightarrow(1+4 \times 5) \bmod 8=5 \\ 6 \rightarrow(1+5 \times 3) \bmod 8=8 & 6 \rightarrow(1+5 \times 5) \bmod 8=2 \\ 7 \rightarrow(1+6 \times 3) \bmod 8=3 & 7 \rightarrow(1+6 \times 5) \bmod 8=7 \\ 8 \rightarrow(1+7 \times 3) \bmod 8=6 & 8 \rightarrow(1+7 \times 5) \bmod 8=4\end{array}$

Figure 4(a) shows the arrangement of bits before interleaving. By applying the new order of column-wise and row-wise interleaved bits obtained from the above calculation and map the positions to get the arrangement of bits after interleaving as shown in Fig. 4(b).

\subsection{FRS Interleaver}

Based on our study for helical and prime interleavers, it is found that the use of $2 \mathrm{D}$-prime interleaver of size $8 \times 8$ with helical interleaver achieves a strong randomization. At the same time, the use of two-stage interleavers is not practical. Therefore, this paper proposes a new interleaver FRS, the first letter of the authors' names that can achieve the same performance of such two interleavers but in one stage.

The concept of the proposed interleaver is as follows:

1. First, arranging the data sequence in the form of a matrix containing $N_{r}$ rows and $N_{c}$ columns provided that each one of them must divides 8 and $N_{r} . N_{c}=L$ where $L$ refers to the number of decoded bits.
Column-Wise

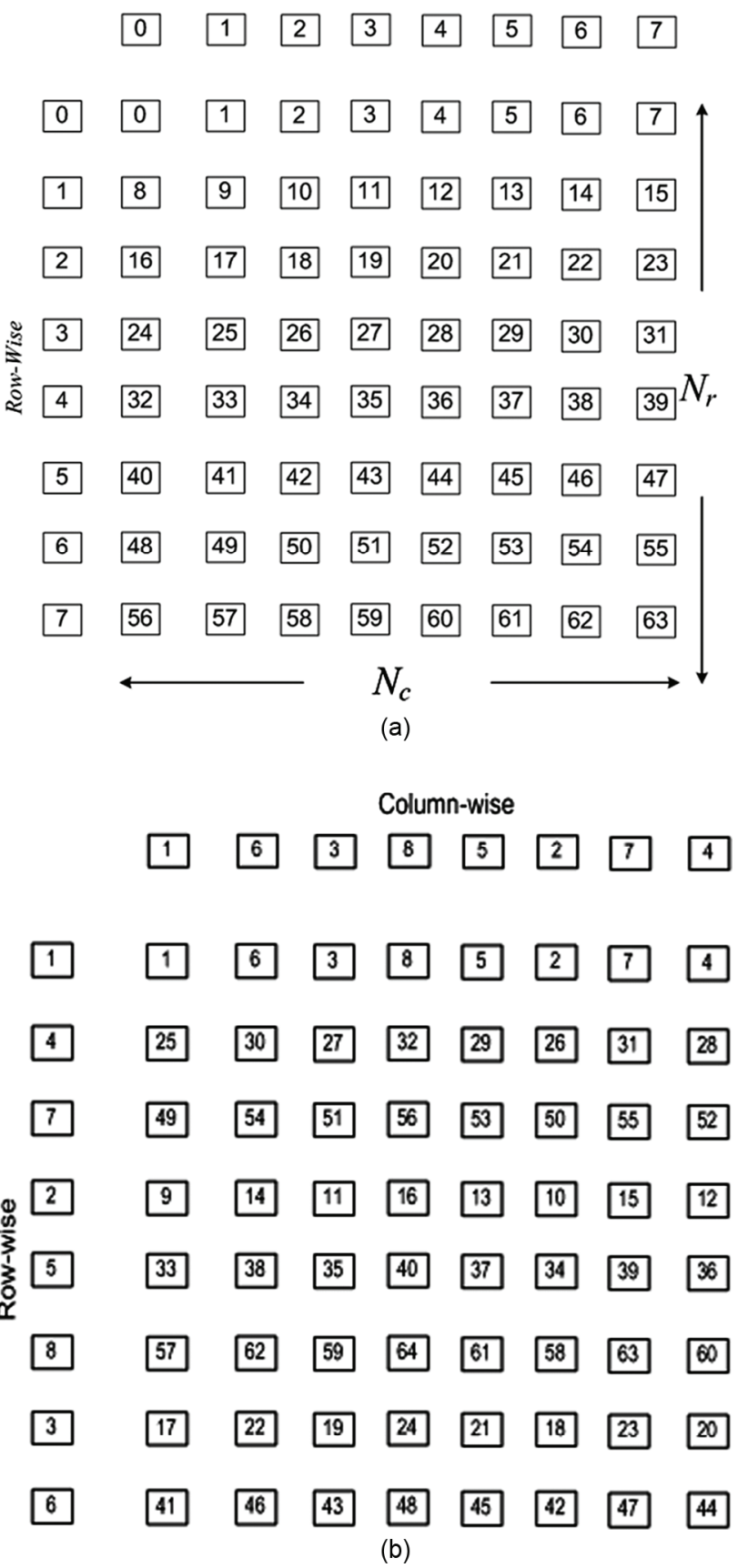

Figure 4. Arrangement of bits of $8 \times 8$ channel: (a) before interleaving and (b) after interleaving.

2. Determine the new location of bits after interleaving using (17) as follows:

$$
\begin{aligned}
i i= & ((1+(i-1) \cdot 3) \bmod 8)+((i-1) \\
& -(i-1) \bmod 8), \quad i=1,2, \ldots, N_{r} \\
j j= & ((1+(j-1) \cdot 5) \bmod 8)+((j-1) \\
& -(j-1) \bmod 8), \quad j=1,2, \ldots, N_{c} \\
\pi[k]= & \pi\left[\left((i i-1)+S \cdot(j j-1) \bmod N_{r}\right)\right. \\
& \left.\cdot N_{c}+j j\right], \quad 0 \leq k<L
\end{aligned}
$$




\begin{tabular}{|c|c|c|c|c|c|c|c|}
\hline$S_{1}$ & $s_{2}$ & $S_{3}$ & $s_{4}$ & $S_{5}$ & $S_{6}$ & $S_{7}$ & $S_{B}$ \\
\hline$S_{g}$ & $S_{10}$ & $S_{11}$ & $s_{12}$ & $S_{13}$ & $S_{14}$ & $S_{15}$ & $S_{16}$ \\
\hline$S_{17}$ & $S_{18}$ & $S_{19}$ & 320 & $s_{21}$ & $s_{22}$ & 82 & $s_{20}$ \\
\hline$s_{\text {工 }}$ & $s_{25}$ & $s_{Z}$ & $s_{\text {工 }}$ & $s_{20}$ & $\$_{30}$ & $s_{31}$ & $\$_{32}$ \\
\hline$S_{33}$ & $S_{34}$ & $S_{35}$ & $S_{36}$ & $S_{37}$ & $\varepsilon_{7}$ & $\delta_{7}$ & $S_{0}$ \\
\hline$S_{41}$ & $S_{42}$ & $S_{43}$ & $S_{44}$ & $S_{45}$ & $S_{45}$ & $S_{47}$ & $3_{40}$ \\
\hline$S_{49}$ & $S_{50}$ & $S_{51}$ & $S_{52}$ & $\varsigma_{53}$ & $S_{54}$ & $\$_{55}$ & $\$_{56}$ \\
\hline$S_{57}$ & $S_{58}$ & $S_{59}$ & $S_{60}$ & $S_{61}$ & $S_{62}$ & $S_{63}$ & $S_{60}$ \\
\hline
\end{tabular}

(a)

\begin{tabular}{|c|c|c|c|c|c|c|c|}
\hline$\$_{1}$ & $S_{54}$ & $\$_{35}$ & $S_{24}$ & $S_{5}$ & $\$_{50}$ & $\$_{39}$ & $s_{20}$ \\
\hline$s_{\boldsymbol{Z}}$ & $S_{14}$ & $\$_{59}$ & $S_{48}$ & $S_{\mathbf{2 9}}$ & $S_{10}$ & $S_{63}$ & $S_{44}$ \\
\hline$S_{49}$ & $S_{38}$ & $S_{19}$ & $S_{8}$ & $S_{53}$ & $S_{34}$ & $s_{\boldsymbol{Z}}$ & $S_{4}$ \\
\hline$S_{g}$ & $S_{62}$ & $\$_{43}$ & $s_{32}$ & $\$_{13}$ & $\$_{58}$ & $S_{47}$ & $s_{\text {Z }}$ \\
\hline$\$_{33}$ & $s_{22}$ & $S_{3}$ & $\$_{56}$ & $S_{37}$ & $\$_{18}$ & $s_{7}$ & $\$_{52}$ \\
\hline$S_{57}$ & $S_{45}$ & $s_{Z}$ & $S_{16}$ & $S_{61}$ & $S_{42}$ & $S_{31}$ & $S_{12}$ \\
\hline$S_{17}$ & $S_{6}$ & $\$_{51}$ & $S_{40}$ & $s_{21}$ & $s_{2}$ & $\$_{55}$ & $s_{36}$ \\
\hline$S_{41}$ & $S_{30}$ & $s_{11}$ & $S_{64}$ & $S_{45}$ & $s_{26}$ & $S_{15}$ & $S_{60}$ \\
\hline
\end{tabular}

(b)

\begin{tabular}{|c|c|c|c|c|c|c|c|}
\hline$S_{1}$ & $S_{2}$ & $S_{3}$ & $S_{4}$ & $\$_{5}$ & $S_{6}$ & $S_{7}$ & $S_{8}$ \\
\hline$S_{9}$ & $S_{10}$ & $S_{11}$ & $S_{12}$ & $S_{13}$ & $S_{14}$ & $S_{15}$ & $S_{16}$ \\
\hline$S_{17}$ & $S_{18}$ & $s_{19}$ & $s_{20}$ & $S_{21}$ & $s_{22}$ & $s_{\boldsymbol{Z}}$ & $S_{24}$ \\
\hline$s_{\text {工 }}$ & $S_{26}$ & $s_{Z Z}$ & $s_{\text {Z }}$ & $s_{29}$ & $S_{30}$ & $S_{31}$ & $S_{32}$ \\
\hline$S_{33}$ & $S_{34}$ & $S_{35}$ & $S_{36}$ & $S_{37}$ & $S_{38}$ & $S_{39}$ & $S_{40}$ \\
\hline$S_{41}$ & $S_{42}$ & $S_{43}$ & $S_{44}$ & $S_{45}$ & $S_{46}$ & $S_{47}$ & $S_{48}$ \\
\hline$S_{49}$ & $s_{50}$ & $\$_{51}$ & $S_{52}$ & $S_{53}$ & $S_{54}$ & $\varsigma_{55}$ & $S_{56}$ \\
\hline$S_{57}$ & $S_{58}$ & $S_{59}$ & $S_{60}$ & $S_{61}$ & $S_{62}$ & $S_{63}$ & $S_{64}$ \\
\hline
\end{tabular}

(c)

Figure 5. Proposed new interleaving of an $8 \times 8$ matrix: (a) the $8 \times 8$ matrix; (b) proposed interleaving of the $8 \times 8$ matrix with $S=2$; and (c) effect of error bursts after de-interleaving.
Where, $S$ is a constant integer. Due to the inherent strong randomization ability of our proposed new interleaver, it can be used for data interleaving efficiently. Figure 5 shows an example of our proposed interleaving of an $8 \times 8$ square matrix and $S=2$. Note that, the helical interleaver can treat both $1 \mathrm{D}$ and $2 \mathrm{D}$ bursts of errors. As a result, a better BER performance can be achieved with the new proposed interleaving mechanism.

\section{CPM-OFDM with FRS}

The main idea of the proposed scheme is to use a combination of CPM-OFDM system with FRS interleaving. The proposed system combines the advantages of frequency diversity and power efficiency from CPM-OFDM and performance improvement from FRS interleaving. The block diagram of the proposed system is shown in Fig. 6. The conventional CPM-OFDM transmitter block is modified by adding an interleaving stage. Both the real in-phase and imaginary quadrature fields of the CPM-OFDM signal are interleaved using the new map. At the receiver, the process is reversed. As the data is processed to the FRS de-interleaving, the receiver is assumed to have an ideal knowledge of the value of $S$, constant integer.

\section{Simulation Results}

All of the experiments in this section are analysed using our Matlab version 7.5 program as optimization toolbox. Experiments are conducted on i5-2.3 GHz laptop running Microsoft Windows 7. In this section, computer simulations were performed to examine and evaluate the new proposed model in Fig. 6. The evaluation starts by BER Performance Evaluation, followed by BER Performance Evaluation for Different Modulation Index, BER Performance Evaluation When RMS Delay Spread, and finally the Overall Complexity Evaluation of the FRS interleaver compared to helical interleaver in terms of hardware requirements.
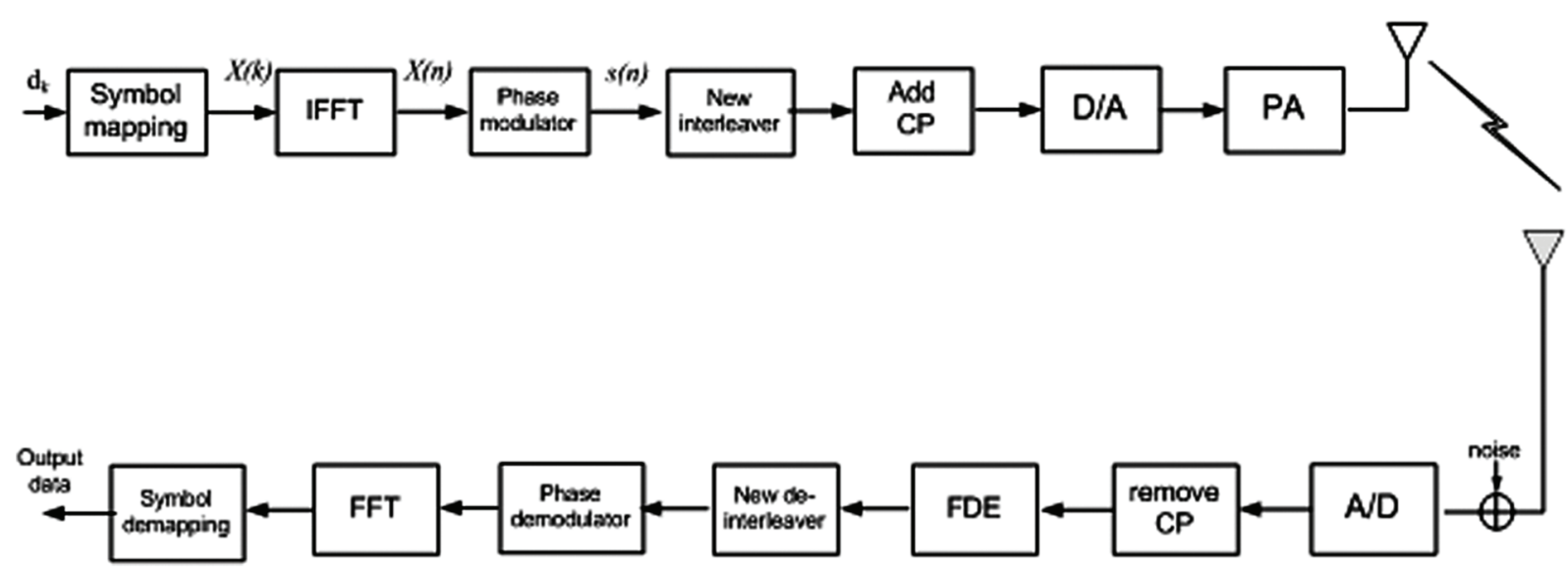

Figure 6. The CPM-OFDM system model with FRS interleaver. 


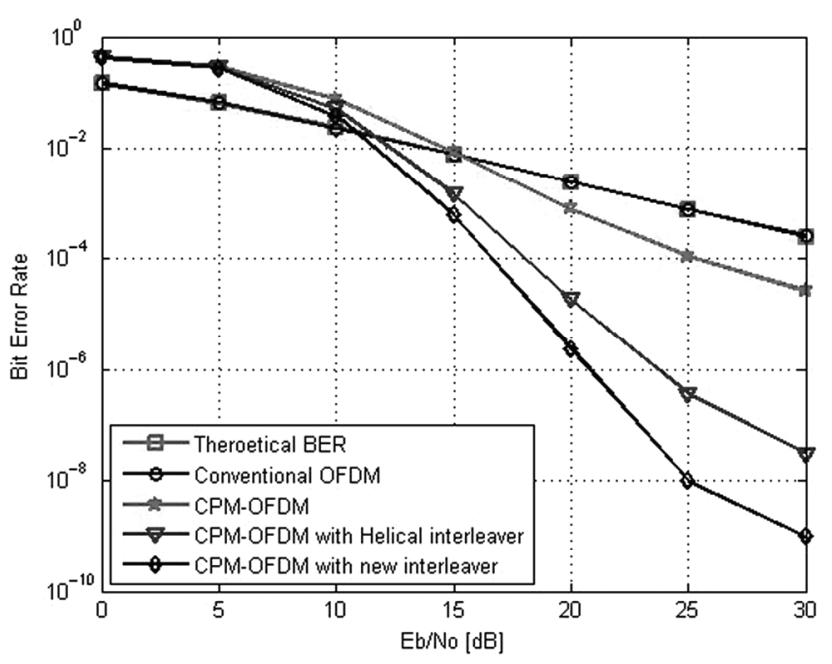

Figure 7. BER performance of the proposed CPM-OFDM with FRS system with new interleaving, the CPMOFDM system without interleaving, and the conventional OFDM at $E_{b} / N_{0}=20 \mathrm{~dB}$ and RMS delay spread $\tau_{\mathrm{rms}}=2 \mu \mathrm{s}$.

\subsection{BER Performance Evaluation}

In this experiment, we evaluate the BER performances of the proposed CPM-OFDM with FRS as a new interleaver. Here, different systems are compared such as follows: (1) theoretical BER, (2) conventional OFDM [40], (3) CPM-OFDM, (4) CPM-OFDM and helical interleaver, and (5) CPM-OFDM with FRS. The comparison is implemented in terms of $E_{b} / N_{0}$ over multipath Rayleigh fading channel model in the absence of AWGN. 4-PAM data symbols are used in this experiment.

As shown in Fig. 7 at $S=5$ and $2 \pi h=1$, it is clear that the proposed CPM-OFDM system with FRS outperforms all other systems. For example, at a $\mathrm{BER}=10^{-3}$, the proposed CPM-OFDM system with FRS interleaving provides $E_{b} / N_{0}$ gains of $3 \mathrm{~dB}, 7 \mathrm{~dB}$, and $12 \mathrm{~dB}$ over CPM-OFDM with helical interleaver, conventional CPM-OFDM system, and the conventional OFDM system, respectively. This is due to the high immunity to burst errors of the proposed system.

\subsection{BER Performance Evaluation for Different Modulation Index at $E_{b} / N_{0}=20 \mathrm{~dB}$}

In this set of experiments, we evaluate the BER performances of the proposed CPM-OFDM with FRS system compared to the conventional CPM-OFDM [40] and OFDM systems in terms of modulation index at $E_{b} / N_{0}=20 \mathrm{~dB}$. The channel model considered is multipath Rayleigh fading channel model with exponential power delay profile. As shown in Fig. 8, it is clear that the proposed CPM-OFDM system outperforms the conventional CPMOFDM system and CPM-OFDM with helical interleaver. That is to say, the new interleaving scheme improves the bandwidth efficiency when used with CPM-OFDM system. For example, at BER $=2.1 \times 10^{-4}$, the proposed CPM-OFDM system needs $2 \pi h=0.7$ and CPM-OFDM

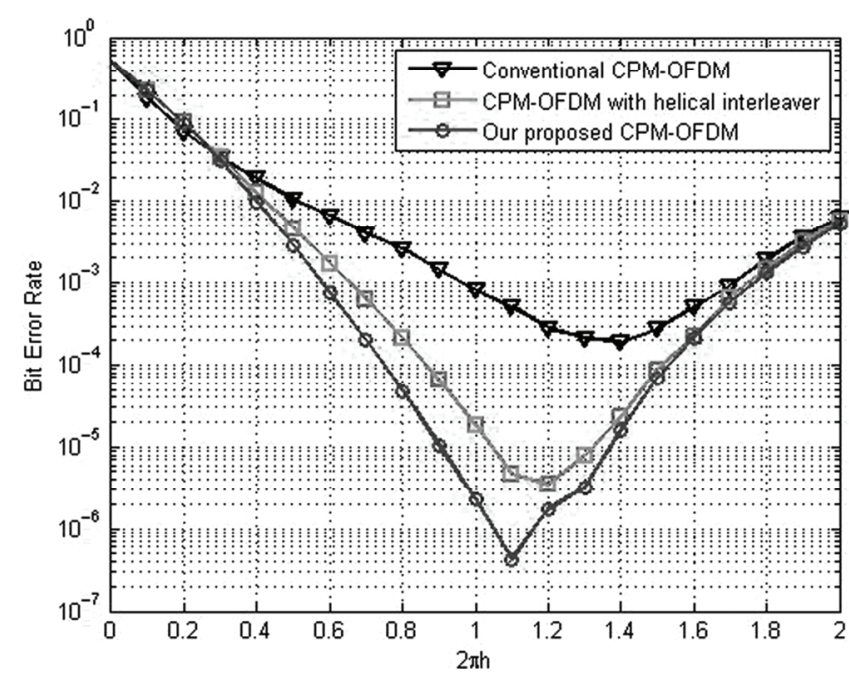

Figure 8. BER performance of the conventional CPMOFDM and the proposed CPM-OFDM systems in terms of $2 \pi h$ at $E_{b} / N_{0}=20 \mathrm{~dB}$.

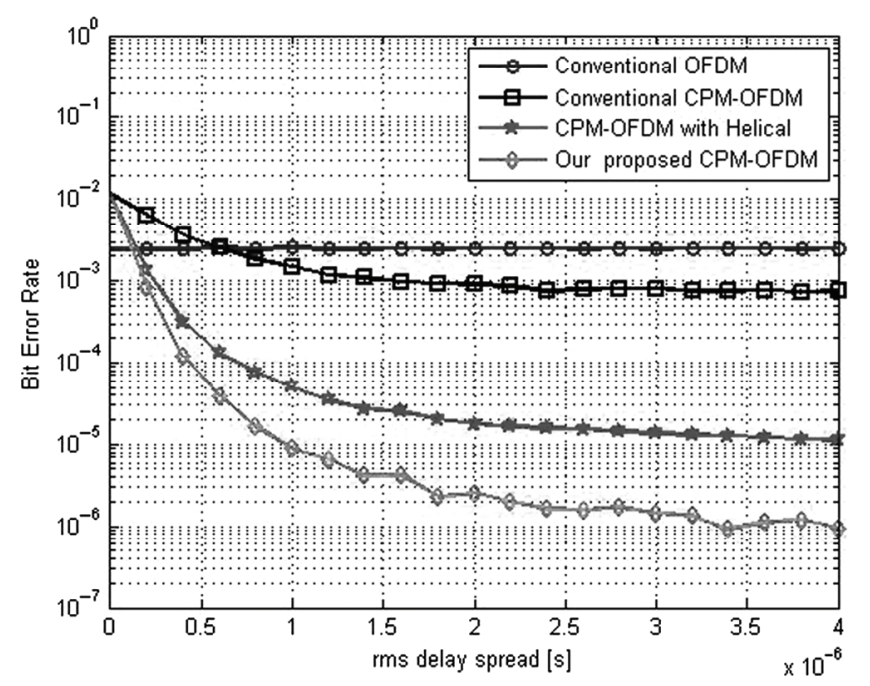

Figure 9. BER performance of the conventional CPMOFDM and the proposed CPM-OFDM with FRS systems in terms of RMS delay spread at $E_{b} / N_{0}=20 \mathrm{~dB}$.

with helical interleaver needs $2 \pi h=0.8$. According to (8), the bandwidth efficiency will be $\eta=2 \mathrm{bps}$ for both. On the other hand, the conventional CPM-OFDM system needs $2 \pi h=1.4$, i.e., $\eta=1.43$ bps. Therefore, we can say that the proposed CPM-OFDM system can achieve about $29 \%$ improvement in bandwidth efficiency when compared to the conventional CPM-OFDM system.

\subsection{BER Performance Evaluation When RMS Delay Spread at $E_{b} / N_{0}=20 \mathrm{~dB}$ and $2 \pi h=1$}

In this set of experiments, we evaluate the BER performances of the proposed CPM-OFDM with FRS system compared to the conventional CPM-OFDM [40] and OFDM systems in terms of RMS delay spread at $E_{b} / N_{0}=20 \mathrm{~dB}$ and $2 \pi h=1$. The channel model considered is multipath Rayleigh fading channel model with exponential power delay profile as shown in Fig. 9, in the flat 


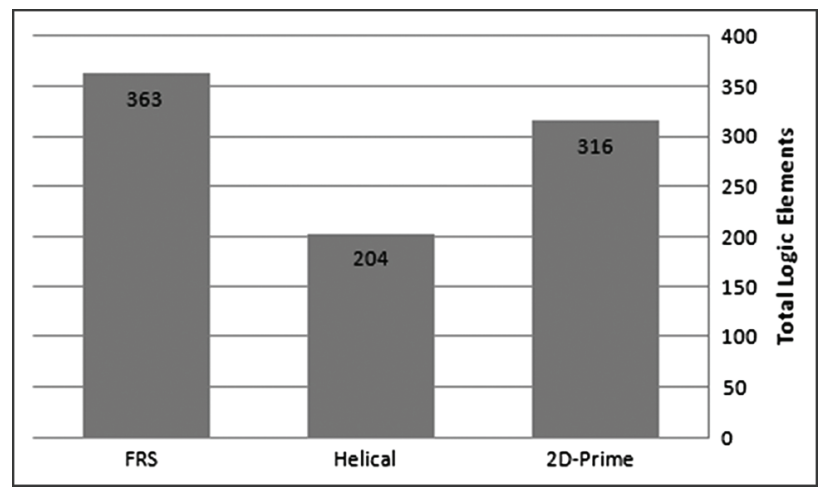

Figure 10. The total logic elements required for the FPGA implementation of FRS, 2D-prime, and helical interleavers of size $128 \times 128$.

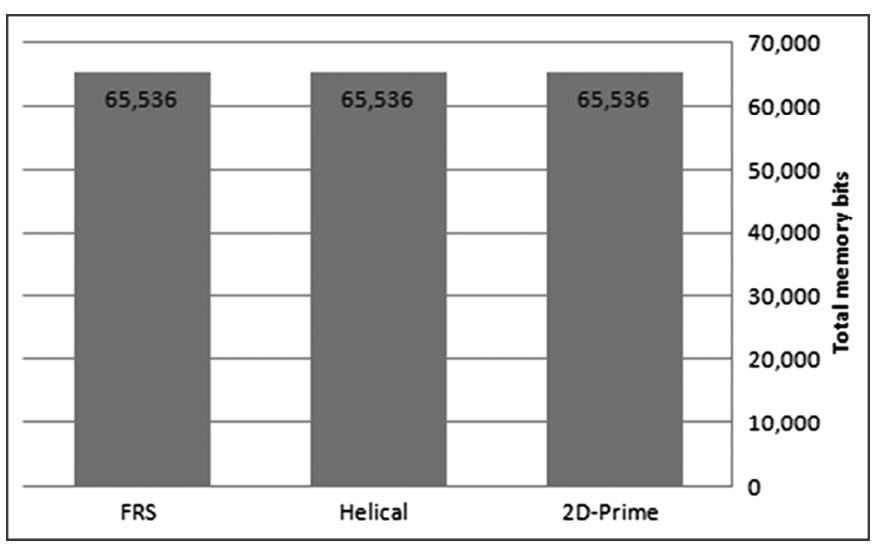

Figure 11. The total memory bits required for the FPGA implementation of FRS, 2D-prime, and helical interleavers of size $128 \times 128$.

fading case (i.e., $\tau_{\mathrm{rms}}=0$ ), the performance of the conventional CPM-OFDM, CPM-OFDM with helical interleaver, and proposed CPM-OFDM systems converge with a small performance loss compared to the conventional OFDM system. This is due to the effect of the phase demodulator threshold. But, in the frequency selective channels $\left(\tau_{r m s}>0\right)$, it is clear that the proposed CPM-OFDM with FRS system outperforms all other systems.

\subsection{Overall Complexity Evaluation}

In this set of experiments, we evaluate the overall complexity of the FRS interleaver compared to helical and 2Dprime interleavers in terms of hardware requirements. The implementation has been carried out in hardware using field programmable gate array (FPGA) for the entire two interleavers of size $128 \times 128$. The FPGA board used is Altera's Cyclone II to achieve the design of the interleavers. We obtain the overall complexity in terms of total logic elements and memory bits as shown in Figs. 10 and 11, respectively. Altera's quartus II tools have been used to estimate the required logic elements and memory bits for the FPGA implementation of FRS, helical, and 2D-prime interleavers. As can be seen in the figures, the FRS interleaver has more logic elements than helical and 2Dprime interleavers while the total memory bits are the same for both interleavers. Therefore, we can say that the FRS interleaver is more complex than helical and 2D-prime interleavers. That is to say, FRS interleaver will require more energy in computation. However, the energy consumption in communication has been identified as the major source of energy consumption and costs significantly more than computation in WSNs. Indeed, increasing the BER will increase the number of lost packets which in turn increases the energy consumption due to the packet retransmission that inevitably affects the network efficiency. Therefore, FRS interleaver can improve the network throughput and energy-efficiency compared with others.

\section{Conclusion}

This paper introduced the concept of using CPM with OFDM for OFDM performance enhancement for wireless networks such as WSNs. However, the CPM-OFDM still suffers from the PAPR problem. Therefore, the paper proposed to use an interleaver to solve this problem. The helical interleaver is utilized and examined. At the same time, a new proposal FRS is presented. The performance of the proposed scheme was studied over multipath Rayleigh fading channel model with exponential power delay spread. The obtained simulation results show a noticeable performance improvement over conventional OFDM, CPMOFDM, and CPM-OFDM with helical interleaver systems, especially at high RMS delay spread. Furthermore, according to the simulation results, it has been observed that a moderate modulation index allows an efficient utilization of the channel frequency diversity, while maintaining high bandwidth efficiency. Finally, the disadvantage of the proposed FRS interleaver is that it is more complex than helical and 2D-prime interleavers.

\section{Acknowledgements}

This work is funded by a grant from the Long-Term National Plan for Science, Technology and Innovation (LT-NPSTI), No. 11-INF1702-10, the King Abdulaziz City for Science and Technology (KACST), Kingdom of Saudi Arabia. We thank the Science and Technology Unit at Umm A-Qura University for their continued logistics support.

\section{References}

[1] M. Ilyas and I. Mahgoub, Handbook of sensor networks: Compact wireless and wired sensing systems (New York, NY: CRC Press, 2005).

[2] R. Amasa, Inter carrier interference cancellation in OFDM systems, M. Tech thesis, Department Electronics and Communication Engineering, National Institute of Technology Rourkela, Rourkela, Orissa, India, 2009.

[3] J. Bingham, Multicarrier modulation for data transmission: an idea whose time has come, IEEE Communications Magazine, 28, 1990, 5-14.

[4] J. Henaut, A. Lecointre, D. Dragomirescu, and R. Plana, Radio interface for high data rate wireless sensor networks, Proc. Int. Conf. on Computing Communications and Control Technologies, Orlando, FL, April 1, 2010. 
[5] S.C. Thompson and A.U. Ahmed, Constant envelope OFDM, IEEE Transactions on Communication, 56(8), 2008, $1300-1312$.

[6] K. Dostert, Power line communications (Upper Saddle River, NJ: Prentice-Hall, 2001).

[7] C.K. Sung, J. Heo, and I. Lee, Adaptive bit-interleaved coded OFDM with reduced feedback information, IEEE Transactions on Communications, 55(9), 2007, 1649-1655.

[8] S.W. Lei and V.K.N. Lau, Performance analysis of adaptive interleaving for OFDM systems, IEEE Transactions on Vehicular Technology, 51(3), 2002, 435-444.

[9] Z. Abdel Hamid, M. Samir, S.M. Abd El-atty, A.E. El-Hennawy, H.El Shenawy, S.A. Alshebeili, and F.E. Abd El-Samie, On the performance of FFT/DWT/DCT-based OFDM systems with chaotic interleaving and channel estimation, Wireless Personal Communication, 78(2), 2014, 1495-1510.

[10] Y.Q. Shi, X.M. Zhang, Z.-C. Ni, and N. Ansari, Interleaving for combating bursts of errors, IEEE Circuits and Systems Magazine, 4, 2004, 29-42.

[11] V.D. Nguyen and H. Kuchenbecker, Block interleaving for soft decision Viterbi decoding in OFDM systems, IEEE VTC, Atlantic City, NJ, USA, 2001, 470-474.

[12] Y. Wu and W.Y. Zou, Orthogonal frequency division multiplexing: A multicarrier modulation scheme, IEEE Transactions on Consumer Electronics, 41(3), 1995, 392-399.

[13] S. Hara and R. Prasad, Multicarrier techniques for $4 G$ mobile communications (Boston: Artech House, 2003).

[14] S.H. Han and J.H. Lee, An overview of peak-to-average power ratio reduction techniques for multicarrier transmission, IEEE Wireless Communications, 12(2), 2005, 56-65.

[15] J.B. Andersson, T. Aulin, and C.-E. Sundberg, Digital phase modulation (New York, NY: Plenum Press, 1986).

[16] J. Henaut, A. Lecointre, D. Dragomirescu, and R. Plana, Radio interface for high data rate wireless sensor networks, Int. Conf. on Computing, Communications and Control Technologies, Orlando, FL, 2008.

[17] S.C. Thompson, J.G. Proakis, and J.R. Zeidler, Constant envelope binary OFDM phase modulation, Proc. 23rd Military Communication Conference, Baltimore, MD, 2003.

[18] S.C. Thompson, A. U. Ahmed, J.G. Proakis, and J.R. Zeidler. Constant envelope OFDM phase modulation: spectral containment, signal space properties and performance, Proc. 23rd Military Communication Conference, Baltimore, MD, 2004.

[19] Tsai, G. Zhang, and J.L. Pan, Orthogonal frequency division multiplexing with phase modulation and constant envelope design, Proc. 23rd Military Communication Conf., Baltimore, MD, 2005.

[20] S.C. Thompson, J.G. Proakis, and J.R. Zeidler, Noncoherent reception of constant envelope OFDM in flat fading channels, Proc. IEEE 16th Int. Symp. on PIMRC, Baltimore, MD, 2005.

[21] S. Benedetto, G. Montorsi, A. Perotti, and A. Tarable, Optimization of CPM pragmatic capacity, IEEE GLOBECOM, Washington, DC, USA, 2007, 1421-1425.

[22] B.E. Rimoldi, A decomposition approach to CPM, IEEE Transactions on Information Theory, 34, 1988, 260-270.

[23] S.C. Thompson and A.U. Ahmed, Constant envelope OFDM, IEEE Transactions on Communication, 56(8), 2008. http://elsteve.com/thesis/

[24] S.C. Thompson, Constant envelope OFDM phase modulation, Ph.D. Dissertation, University of California, San Diego, 2005. [Online]. Available: http://elsteve.com/thesis/

[25] M.K. Simon and M.-S. Alouini, Digital communication over fading channels (New York, NY: John Wiley \& Sons, Inc., 2000).

[26] J.G. Proakis and M. Salehi, Communication systems engineering (Upper Saddle River, NJ: Prentice Hall, 1994).

[27] M. Pätzold, Mobile fading channels (West Sussex, England: John Wiley \& Sons, 2002).

[28] E.S. Hassan, X. Zhu, S.E. El-Khamy, M.I. Dessouky, S.A. El-Dolil, and F.E.A. El-Samie, A continuous phase modulation single-carrier wireless system with frequency domain equalization, Proc. IEEE Int. Conf. Computer Engineering 85 Systems (ICCES), Cairo, Egypt, 2009, 599-604.

[29] H. Sari, G. Karam, and I. Jeanclaude, Transmission techniques for digital terrestrial TV broadcasting, IEEE Communications Magazine, 3, 1995, 100-109.
[30] Y. Tsai, G. Zhang, and J.-L. Pan, Orthogonal frequency division multiplexing with phase modulation and constant envelope design, Proc. IEEE Milcom, 4, 2005, 2658-2664.

[31] S. Hara and R. Prasad, Overview of multicarrier CDMA, IEEE Communication Magazine, 35, 1997, 126-144.

[32] E.S. Hassa, Xu Zhu, S.E. El-Khamy, et al., Enhanced performance of OFDM and single-carrier systems using frequency domain equalization and phase modulation, 26th National Radio Science Conference (NRSC2009), Cairo, Egypt, 2009, 1-10.

[33] J.G. Proakis and D.G. Manolakis, Digital signal processing: principles, algorithms, and applications, 3rd ed. (Upper Saddle River, NJ: Prentice Hall, 1996).

[34] E.S. Hassan, S.E. El-Khamy, M.I. Dessouky, S.A. El-Dolil, and F.E. Abd El-Samie, New interleaving scheme for continuous phase modulation based OFDM systems using chaotic maps, Proc. of WOCN-09, Cairo, Egypt, 2009, 28-30.

[35] T.S. Rappaport, Wireless communications principles $\& 5$ practice, (Upper Saddle River, NJ: Prentice-Hall, 1998).

[36] J.G. Proakis, Digital communication, 4th ed. (Boston: McGraw-Hill, 2001).

[37] R. Bansal, S. Anand, D. Purwar, and A. Shukla, Application of antenna diversity in HIDMA scheme using gold codes, Proc. of IEEE Int. Conf. on Communication System and Network Technologies, Katra, Jammu, 2011, 228-231.

[38] D. Hao and P.A. Hoeher, Helical interleaver set design for interleave-division multiplexing and related techniques, IEEE Communications Letters, 12(11), 2008, 843-845.

[39] P. Hanpinitsak and C. Charoenlarpnopparut, 2D interleaver design for image transmission over severe burst-error environment, International Journal of Future Computer and Communication, 2(4), 2013, 308-312.

[40] E.S. Hassan, Xu Zhu, S.E. El-Khamy, M.I. Dessouky, S.A. El-Dolil, and F.E. Abd El-Samie, Performance evaluation of OFDM and single carrier systems using frequency domain equalization and phase modulation, International Journal of Communication Systems, 24, 2011, 1-13.

\section{Biographies}

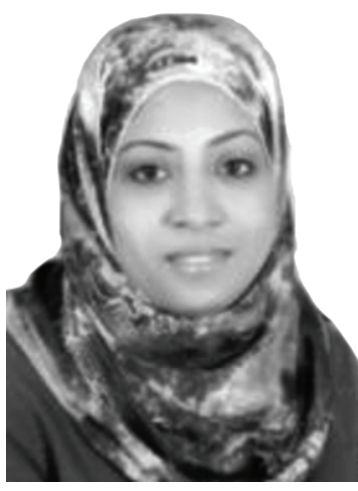

Fatma H. El-Fouly received her B.S.(Hons.) and M.S. degrees in electronic engineering from Menoufia University, Menouf, Egypt, in 2003 and 2008, respectively. Currently, she is working as an instructor at the Department of Electronics and Electrical Communication, Higher Institute of Engineering, El-Shorouk Academy. Her current research interests include wireless sensor networks and digital communication fields.

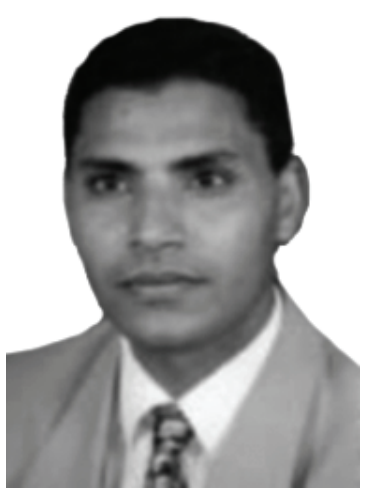

Rabie A. Ramadan is currently an assistant professor at Cairo University. He received his Ph.D. from Southern Methodist University, Dallas, TX, USA, in 2007. His field of expertise is in mobile computing, networking and computational intelligence. In 2005, he joined Banc of America Securities Company in Frisco, Dallas, TX, USA, as a senior developer. The company is part of the Bank of America in which its responsibility is to implement all of 
the bank required software. He was involved in the implementation of many projects such as handling the real-time stock market information and pushing it efficiently to different customers. He was one of the founders of "Ibda" company in Egypt as Software Company from the 1995 to 2003 where he was serving as the head of the web programming department. He has also great experience in dealing with networks where he was working as a network administrator at International Telecommunication Union (ITU) regional office. He is a cofounder of the IEEE Computational Intelligence Egypt chapter. Currently he is serving as the chapter chair.

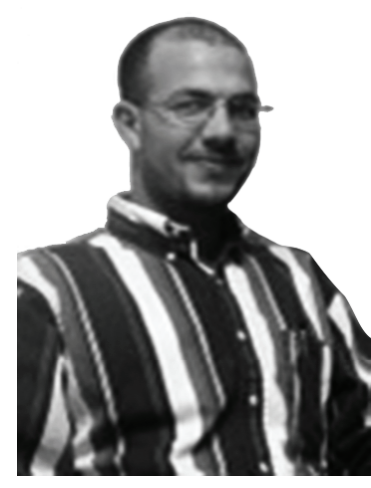

Salah A. Aly completed his Ph.D. in Computer Science from Faculty of Engineering at Texas A\&M University in January 2008. He worked in a research internship at Bell-Labs \& Alcatel-Lucent in New Jersey. He also worked as a research associate at Princeton University in New Jersey. Then, he moved as an assistant professor to Umm Al-Qura University in Mecca. Currently, he is an associate professor at Alfayoum University. His research areas include quantum and classical information processing particularly in the areas of error control codes, algorithms, network coding and information security including their applications in wireless sensor networks and storage systems. 